LBNL-45743

\title{
Photoproduction of top quarks in peripheral heavy ion collisions
}

\author{
Spencer R. Klein ${ }^{1}$, Joakim Nystrand ${ }^{1 *}$, and Ramona $\operatorname{Vogt}^{1,2}$ \\ ${ }^{1}$ Lawrence Berkeley National Laboratory, Berkeley, CA 94720 \\ ${ }^{2}$ Physics Department, University of California, Davis, CA 95616
}

\begin{abstract}
In relativistic heavy ion collisions, top quarks can be produced by photongluon fusion when a photon from the Weizsäcker-Williams virtual photon field of one nucleus interacts with a gluon in the other nucleus. Photoproduction with heavy ions at the Large Hadron Collider (LHC) will be the first accessible non-hadronic top production channel. We calculate the $t \bar{t}$ photoproduction cross sections, pair mass and top quark rapidity distributions in peripheral lead-lead and oxygen-oxygen collisions. The cross sections are sensitive to the top quark charge and the large- $Q^{2}$ gluon distribution in the nucleus. We find a cross section of $15 \mathrm{pb}$ in oxygen-oxygen collisions, leading to 210 pairs in a one month $\left(10^{6} \mathrm{~s}\right)$ LHC run. In $p A$ collisions, the rate is higher, 1100 pairs per month for $p \mathrm{O}$. A comparison of the $A A$ and $p A$ data might allow for a study of gluon shadowing at high $Q^{2}$.
\end{abstract}

Typeset using REVTEX

*Present address: Division of Cosmic and Subatomic Physics, Department of Physics, Lund University, Lund SE-22100, Sweden 
Due to their large charges, relativistic heavy ions carry strong electromagnetic fields which may be treated as virtual photon fields. In a relativistic ion collider, these fields interact with target nuclei in the opposing beam, resulting in high luminosities for photonuclear interactions. Because of the large Lorentz boosts, high photon-nucleon center of mass energies are reached. Previous studies have considered photoproduction of charm [1] and bottom [2] quarks as well as nuclear breakup [3] and vector meson production [4]. These calculations all considered peripheral collisions, with impact parameter, $b$, greater than twice the nuclear radius $R_{A}$ so that the two nuclei do not interact hadronically. Peripheral collisions will be studied experimentally at RHIC [5] and the LHC [6], now under construction at CERN.

Here, we consider the production of top quarks via photon-gluon fusion, paralleling previous calculations of photoproduction in heavy ion collisions [1]2]. We calculate top production from the heaviest, $\mathrm{Pb}$, and lightest, $\mathrm{O}$, ions planned for the LHC. In these collisions, a $t \bar{t}$ is produced in the reaction $\gamma(k)+A(P) \rightarrow t\left(p_{1}\right)+\bar{t}\left(p_{2}\right)+X$ where $k$ is the four momentum of the photon emitted from the virtual photon field of the projectile nucleus, $P$ is the four momentum of the interacting nucleon in target nucleus $A$, and $p_{1}$ and $p_{2}$ are the four momenta of the produced $t$ and $\bar{t}$ quarks. Note that a phton from the target can also interact with a nucleon in the projectile. We work in the center of mass (lab) frame. The photons are almost real, with $\left|q^{2}\right|<\left(\hbar c / R_{A}\right)^{2}$. The slight virtuality is neglected.

On the parton level, the photon-gluon fusion reaction is $\gamma(k)+g\left(x_{2} P\right) \rightarrow t\left(p_{1}\right)+\bar{t}\left(p_{2}\right)$ where $x_{2}$ is the fraction of the target momentum carried by the gluon. To lowest order (LO), the $t \bar{t}$ production cross section is [7]

$$
s^{2} \frac{d^{2} \sigma}{d t_{1} d u_{1}}=\pi \alpha_{s}\left(Q^{2}\right) \alpha e_{t}^{2}\left(\frac{t_{1}}{u_{1}}+\frac{u_{1}}{t_{1}}+\frac{4 m^{2} s}{t_{1} u_{1}}\left[1-\frac{m^{2} s}{t_{1} u_{1}}\right]\right) \delta\left(s+t_{1}+u_{1}\right)
$$

where the partonic invariants $s, t_{1}$, and $u_{1}$ are defined as $s=\left(k+x_{2} P\right)^{2}, t_{1}=(k-$ $\left.p_{1}\right)^{2}-m^{2}=\left(x_{2} P-p_{2}\right)^{2}-m^{2}$, and $u_{1}=\left(x_{2} P-p_{1}\right)^{2}-m^{2}=\left(k-p_{2}\right)^{2}-m^{2}$ with top quark mass $m=175 \mathrm{GeV}$. In this case, $s=4 k \gamma x_{2} m_{p}$ where $\gamma$ is the Lorentz boost of a single beam and $m_{p}$ is the proton mass. Here, $e_{t}=2 / 3$ is the expected top quark charge, $\alpha=e^{2} / \hbar c$ is the electromagnetic coupling constant, and $\alpha_{s}\left(Q^{2}\right) \approx 0.11$ is the one-loop strong 
coupling constant evaluated at scale $Q^{2}=m^{2}+p_{T}^{2}$ where $p_{T}$ is the transverse momentum of the produced top quark. The large mass of the top quark prevents toponium production, allowing the $t$ and $\bar{t}$ to be treated as free quarks, even near threshold [8].

The hadronic top production cross section is obtained by integrating Eq. (11) over $x_{2}$ and $k$, since virtual photons are emitted by the nucleus in a continua of four-momenta,

$$
S^{2} \frac{d^{2} \sigma_{\gamma A \rightarrow t \bar{t} X}}{d T_{1} d U_{1}}=2 \int_{k_{\min }}^{\infty} d k \frac{d N}{d k} \int_{x_{2_{\min }}}^{1} \frac{d x_{2}}{x_{2}} g\left(x_{2}, Q^{2}\right) s^{2} \frac{d^{2} \sigma}{d t_{1} d u_{1}}
$$

where $d N / d k$ is the photon flux. The factor of two in Eq. (2) arises because both nuclei emit photons and thus serve as targets. The incoherence of top production guarantees that there is no interference between the two production sources [9]. The hadronic invariants can be defined for a given photon four momuntum $k$. If the top quark is detected, the invariants are $S=(k+P)^{2}, T_{1}=\left(P-p_{1}\right)^{2}-m^{2}$, and $U_{1}=\left(k-p_{1}\right)^{2}-m^{2}$ 10. The partonic and hadronic invariants are related by $s=x_{2} S, t_{1}=U_{1}$, and $u_{1}=x_{2} T_{1}$. Four-momentum conservation at leading order gives $x_{2_{\min }}=-U_{1} /\left(S+T_{1}\right)$. In addition to the total cross sections, we also present the top quark rapidity distribution and the $t \bar{t}$ invariant mass distribution. We define the $t$ rapidity as $y$ and the $\bar{t}$ rapidity as $y_{2}$. The top quark rapidity is related to the invariant $T_{1}$ by $T_{1}=-\sqrt{S} Q e^{-y}$. The invariant mass of the pair can be determined if both the $t$ and $\bar{t}$ are detected. The invariant mass is $s=M^{2}=2 Q^{2}\left(1+\cosh \left(y-y_{2}\right)\right)$. The minimum photon momentum necessary to produce a $t \bar{t}$ pair is $k_{\min }=M^{2} /\left(4 \gamma x_{2} m_{p}\right)$.

We use the MRST LO gluon distribution $x g\left(x, Q^{2}\right)$ [11] which is considerably softer than the older, flat parameterizations such as $x g(x) \propto(1-x)^{n}$ [12] used in earlier photoproduction predictions. Calculations with $n=5$ result in cross sections $2-3$ times higher than those with the MRST LO gluon distribution. Shadowing, the modification of the gluon distribution in nuclei, is included via a parameterization based on a fit to data [13]. There are two caveats regarding shadowing. First, the large $Q^{2}$ of top production is outside the upper limit of the parameterization, $Q_{\max }^{2}=10^{4} \mathrm{GeV}^{2}$. However, the shadowing parameterization continues to evolve beyond this point with changes on the percent level between $Q^{2}=10^{4} \mathrm{GeV}^{2}$ and $6.4 \times 10^{5} \mathrm{GeV}^{2}$. Secondly, the probable impact parameter dependence of the shadowing 14 
has been neglected.

The photon flux is given by the Weizsäcker-Williams formulae. The flux is a function of the distance from the nucleus, $r$,

$$
\frac{d^{3} N}{d k d^{2} r}=\frac{Z^{2} \alpha w^{2}}{\pi^{2} k r^{2}}\left[K_{1}^{2}(w)+\frac{1}{\gamma^{2}} K_{0}^{2}(w)\right]
$$

where $w=k r / \gamma$ and $K_{0}(w)$ and $K_{1}(w)$ are modified Bessel functions. The photon flux is cut off at an energy determined by the size of the nucleus. In the rest frame of the target nucleus, the cutoff is boosted to $\left(2 \gamma^{2}-1\right) \hbar c / R_{A}$, or $500 \mathrm{TeV}$ for lead and $1800 \mathrm{TeV}$ for oxygen. The $t \bar{t}$ production cross section, $\gamma p \rightarrow t \bar{t} \llbracket 12$, for a photon with the cutoff energy of oxygen is a factor of 7.2 times larger than the cross section at the cutoff energy of lead. Thus the difference in the energy cutoffs of lead and oxygen is significant.

The total photon flux striking the target nucleus is the integral of Eq. (3) over the transverse area of the target at all impact parameters subject to the constraint that the two nuclei do not interact hadronically [1,4]. The numerical result agrees to within $15 \%$ of the analytical result,

$$
\frac{d N}{d k}=\frac{2 Z^{2} \alpha}{\pi k}\left[w_{R} K_{0}\left(w_{R}\right) K_{1}\left(w_{R}\right)-\frac{w_{R}^{2}}{2}\left(K_{1}^{2}\left(w_{R}\right)-K_{0}^{2}\left(w_{R}\right)\right)\right]
$$

the integral of Eq. (3) for $r>2 R_{A} \approx 2.4 A^{1 / 3}$ where $w_{R}=2 k R_{A} / \gamma$. The $15 \%$ difference can serve as a conservative estimate of the uncertainty on the photon flux; this can be checked using other photoproduction and two-photon interactions.

Although our $t \bar{t}$ calculation is at leading order, the large mass of the top quark ensures faster convergence of the perturbative expansion than for the lighter charm and bottom quarks where the next-to-leading order corrections lead to factors of $2-3$ enhancements over the LO cross section. The next-to-leading order $t \bar{t}$ cross sections are only $\approx 40 \%$ larger than the LO cross sections in $p p$ interactions [15]. We assume the NLO enhancement to be similar for photoproduction.

The expected center of mass energies and average luminosities for $\mathrm{O}+\mathrm{O}$ and $\mathrm{Pb}+\mathrm{Pb}$ collisions at the LHC are given in Table [1. The numbers are taken from the latest LHC 
machine studies on LHC ion operation [16]. The calculation of the average luminosities assumes two experiments, e.g. ALICE and CMS, and a bunch spacing of $125 \mathrm{~ns}$.

The total cross sections for $t \bar{t}$ pair production with lead and oxygen beams are $550 \mathrm{pb}$ and $15 \mathrm{pb}$. As Table 1 shows, a $10^{6} \mathrm{~s}$ (one month) heavy ion run at design luminosity will produce 0.2 and 210 pairs respectively. The most significant factor in the rate difference comes from the luminosity, $\sim 3 \times 10^{4}$ times higher for oxygen.

Shadowing plays little role in the total cross sections since at $y=0$ and $p_{T}=0,\left\langle x_{2}\right\rangle \approx$ 0.06 for lead and 0.05 for oxygen. These values are in a region where shadowing effects are small. In addition, at the large $Q^{2}$ required for top production, shadowing effects are further reduced by $Q^{2}$ evolution [13]. The uncertainty in the large- $Q^{2}$ shadowing is important since no data are available. However, because of the large luminosity gain, lighter mass ions are better for studies of nuclear gluon distributions, reducing any possible effect still further.

The top quark rapidity distributions, obtained from Eq. (2), are shown in Fig. 1. The calculations assume that the photon is in the field of the nucleus coming from positive rapidity so that $y<0$ corresponds to $k<\gamma x_{2} m_{p}$ in the center of mass (lab) frame. If the photon is emitted by the target instead of the projectile, the resulting top quark rapidity distribution would then be the mirror image of the distribution in Fig. 1 around $y=0$. (Note that this mirror distribution is equivalent to detecting the $\bar{t}$ instead of the $t$. Thus the $t$ and $\bar{t}$ distributions are not symmetric around $y=0$.) The total top quark rapidity distribution is the sum of the curve in Fig. 1 with its mirror image when both nuclei emit photons. Roughly half the production is within $|y|<1$, in the central ALICE acceptance and almost all $t \bar{t}$ production falls within the CMS acceptance, $|y|<2.4$. The $t \bar{t}$ pair invariant mass distributions, $d \sigma / d M$, are shown in Fig. 2. The larger photon energy of oxygen results in a broader pair mass distribution.

The only previous study of $t \bar{t}$ production via photon-gluon fusion in heavy ion collisions [17] used a very different photon flux. The flux was integrated over all $r$, including the nuclear interior, modelling the nucleus as a homogenously charged sphere. This approach is flawed because the photon flux inside the nucleus is poorly defined. Ref. [18] shows that 
the photon flux varies by orders of magnitude inside the nucleus, depending on the chosen nuclear form factor. More importantly, the calculation is incorrect because the photon flux inside the nucleus is much higher than that outside the nucleus. The authors apply a correction factor of $1 / 2$ but this factor is inappropriately large. Their photon flux (and corresponding cross section) for $\mathrm{Pb}+\mathrm{Pb}$ collisions is a factor of 10 greater than ours.

Top quark pairs can be observed via their decays, predominantly $t \bar{t} \rightarrow W^{+} b W^{-} \bar{b}$, where the $W$ decays to $\ell \nu$ or $q \bar{q}^{\prime}$. The major background to these channels is likely to be hadroproduction of top in grazing peripheral collisons with slightly smaller impact parameter. The two reactions can be separated based on the presence of rapidity gaps in the collision, the breakup of the colliding nuclei, and a small multiplicity difference. Because the photon is colorless, even if the nucleus breaks up, photoproduction events should also have a rapidity gap between the photon-emitting nucleus and the $t \bar{t}$ system. The average multiplicity in $p p$ collisions at the LHC is expected to be about 45, slightly higher than the roughly 35 particles expected in a $\gamma p$ collision at typical top-production energies [19. The photoproduction multiplicity should be further reduced since much of the photon energy is needed to produce the $t \bar{t}$ pair, leaving less energy for produce additional particle production.

For lead, nuclear breakup can be a complication. A lead nucleus may be excited into a giant dipole resonance with probability $\approx 35 \% \cdot\left(b / 2 R_{A}\right)^{2}$. When the resonance decays, the nucleus will emit one or more neutrons [20]. To a good approximation, the excitation and the photon-gluon fusion occur independently. Thus the two processes factorize and can be studied separately 21]. For oxygen, the excitation probability is very small, so one of the interacting ions will almost always remain intact.

The photoproduction cross sections are large compared with the corresponding $p p \rightarrow t \bar{t} X$ cross sections. At lowest order, $\sigma(p p \rightarrow t \bar{t} X)=41 \mathrm{pb}$ at $\sqrt{s}=5.5 \mathrm{TeV}, 7 \%$ of the lead photoproduction cross section, while $\sigma(p p \rightarrow t \bar{t} X)=80 \mathrm{pb}$ at $\sqrt{s}=7 \mathrm{TeV}, 5.3$ times the corresponding oxygen photoproduction cross section. Thus, only a moderate hadronic rejection factor is required.

Other backgrounds should be small. Hadronic single or double diffractive production 
without accompanying colored interactions, $A A \rightarrow A A \bar{t} t X$ occurs only in a narrow range of impact parameters. Diffractive production is also suppressed by the $1 / M^{4}$ final-state mass dependence. Any single diffractive top production will be at larger rapidities than photoproduction which is more central. We have calculated $\sigma(\gamma \gamma \rightarrow t \bar{t})$ and found it to be negligible. Backgrounds from other photoproduction channels should be significantly smaller than at hadron colliders because $\sigma(\gamma p \rightarrow Q \bar{Q} X) / \sigma(\gamma p \rightarrow X)$ is much larger than $\sigma(p p \rightarrow Q \bar{Q} X) / \sigma(p p \rightarrow X)$ at comparable energies.

We believe that these criteria should allow for at least statistical separation of events containing photoproduction of top in oxygen on oxygen collisions.

The top photoproduction cross sections are also measurable in $p A$ collisions, as may be possible at the LHC [6.16]. Because the proton and the ion must have the same magnetic rigidity and $Z=A$ for the proton, these collisions are at somewhat higher per nucleon energies than the corresponding $A A$ collisions. The cost is that the center of mass is no longer at rest in the lab. Our $p A$ results are calculated in the equal speed system so that the $\gamma$ is that of the equal speed system. In this case, the photon flux is calculated using the analytical expression in Eq. (4) with $w_{R}=\left(r_{p}+R_{A}\right) k / \gamma$ and $r_{p}=0.6 \mathrm{fm}$ is the proton radius.

The expected nucleon-nucleon center-of-mass energies and luminosities for $p A$ collisions are shown in Table [1. No official LHC $p A$ luminosities are yet available 22]. The values in Table [1] are based on estimates in Ref. [23], which were obtained assuming a 125 ns bunch spacing and one experiment.

The $p A$ collisions would allow a measurement of the gluon structure function in free protons. A comparison between photoproduction in $p A$ and $A A$ collisions at the same energy would provide a straightforward measurement of nuclear gluon shadowing at $Q^{2}$ values far above those currently available. The rates are much higher at the maximum $p A$ energies, however, since the cross sections per nucleon are larger than in $A A$ collisions. The center-of-mass energy is $40-60 \%$ higher, leading to a larger boost in the target rest frame, increasing the photon cutoff energy to $1200 \mathrm{TeV}$ for lead, more than a factor of two greater 
than in $\mathrm{Pb}+\mathrm{Pb}$, and $3600 \mathrm{TeV}$ for oxygen. In addition, the average photon flux is higher in $p A$ because single protons can approach the photon-emitting nucleus more closely than a proton in the center of another nucleus. The estimated luminosities for $p A$ interactions lead to 40 pairs in $p \mathrm{~Pb}$ and 1100 pairs in $p \mathrm{O}$ over a $10^{6} \mathrm{~s}$ run, as shown in Table $[\mathrm{II}$.

Although the top quarks are produced inside the target nucleus, because of their very high boost with respect to the target, they decay well outside the nucleus. For a standard model top width of $\Gamma_{t}=1.5 \mathrm{GeV}$ [24] the top quarks typically travel over $100 \mathrm{fm}$ in the nuclear rest frame before decaying. Thus the $t \bar{t}$ pair acts as a dipole with separation $\hbar c / m \sim 10^{-3} \mathrm{fm}$, resulting in a small interaction cross section. Therefore, aside from debris from the target nucleon, the target nucleus will be relatively undisturbed, leaving the $t \bar{t}$ pair with relatively few accompanying particles.

In conclusion, top quark pairs will be produced via photon-gluon fusion in heavy ion collisions at the LHC. Heavy ion photoproduction will be the first accessible non-hadronic production channel for $t \bar{t}$ pairs. A $10^{6} \mathrm{~s} \mathrm{O}+\mathrm{O}$ run will produce $210 t \bar{t}$ pairs while $p A$ runs will result in higher rates, up to 1100 pairs in $p \mathrm{O}$ collisions. These events could at least be statistically identifiable on the basis of accompanying rapidity gaps, the presence of an intact nucleus, and a slightly smaller multiplicity. The data should allow for measurements of the top charge and mass. In addition, the nuclear gluon distribution may be measurable at large $Q^{2}$.

This work was supported in part by the Division of Nuclear Physics of the Office of High Energy and Nuclear Physics of the U. S. Department of Energy under Contract No. DE-AC-03-76SF00098. 


\section{REFERENCES}

[1] N. Baron and G. Baur, Phys. Rev. C 48, 1999 (1993).

[2] M. Greiner, M. Vidović, C. Hoffman, A. Schäfer and G. Soff, Phys. Rev. C 51, 911 (1995); Ch. Hoffman, G. Soff, A. Schäfer and W. Greiner, Phys. Lett. B262, 210 (1991).

[3] G. Baur, K. Hencken and D. Trautman, J. Phys. G 24, 1457 (1998).

[4] S.R. Klein and J. Nystrand, Phys. Rev. C 60, 014903 (1999).

[5] S. Klein, nucl-ex/0104016, to appear in Proc. 17th Winter Workshop on Nuclear Dynamics, Park City, USA, Mar. 10-17, 2001; J. Nystrand and S. Klein, nucl-ex/9811007, in Proc. Workshop on Photon Interactions and the Photon Structure, eds. G. Jarlskog and T. Sjöstrand, Lund, Sweden, September 1998.

[6] E. Lippmaa et al., FELIX: A full acceptance detector at the LHC, CERN/LHCC 97-45, August, 1997.

[7] L.M. Jones and H.W. Wyld, Phys. Rev. D 17, 759 (1978).

[8] I. Bigi, Y. Dokshitzer, V. Khoze, J. Kühn and P. Zerwas, Phys. Lett. B181, 157 (1986).

[9] S.R. Klein and J. Nystrand, Phys. Rev. Lett. 84, 2330 (2000).

[10] J. Smith and W.L. van Neerven, Nucl. Phys. B374, 36 (1992).

[11] A.D. Martin, R.G. Roberts, W.J. Stirling and R.S. Thorne, Eur. Phys. J. C4, 463 (1998); Phys. Lett. B 443, 301 (1998).

[12] H. Fritzsch and K.H. Streng, Phys. Lett. B 72, 385 (1978).

[13] K.J. Eskola, V.J. Kolhinen and P.V. Ruuskanen, Nuc. Phys. B535, 351 (1998); K.J. Eskola, V.J. Kolhinen and P.V. Ruuskanen, Eur. Phys. J. C9, 61 (1999).

[14] V. Emel'yanov, A. Khodinov, S.R. Klein and R. Vogt, Phys. Rev. C 61, 044904 (2000); V. Emel'yanov, A. Khodinov, S.R. Klein and R. Vogt, Phys. Rev. Lett. 81, 1801 (1998). 
[15] M.L. Mangano, P. Nason, and G. Ridolfi, Nucl. Phys. B373 (1992) 295.

[16] D. Brandt, LHC Project Report 450, December, 2000.

[17] S.M. Schneider, W. Greiner and G. Soff, Phys. Rev. D 46, 2930 (1992).

[18] M. Vidovic, M. Greiner, C. Best and G. Soff, Phys. Rev. C 47, 2308 (1993).

[19] D.E. Groom et al., Eur. Phys. J. C15, 1 (2000).

[20] K. Hencken, D. Trautmann and G. Baur, Z. Phys. C68, 473 (1995).

[21] M.C. Abreu et al. (NA50 Collab.), Phys. Rev. C 59, 876 (1999).

[22] D. Brandt, private communication.

[23] A. Morsch, ALICE Note 97-13, July, 1997.

[24] M. Mangano and T. Trippe, Eur. Phys. J. C3, 343 (1998). 


\section{TABLES}

\begin{tabular}{lcccc}
\hline \hline Ion Species & $\begin{array}{c}\sqrt{S_{N N}} \\
(\mathrm{TeV})\end{array}$ & $A A$ Luminosity & $\sigma(A A)$ & Rate \\
& 7.0 & $1.4 \times 10^{-5}$ & $(\mathrm{pb})$ & (per month) \\
\hline Oxygen & 5.5 & $4.2 \times 10^{-10}$ & 15 & 210 \\
Lead & & 550 & 0.2 \\
\hline \hline
\end{tabular}

TABLE I. Center of mass energies per nucleon-nucleon collision, $\sqrt{S_{N N}}$, and luminosities for heavy ion collisions at the LHC. The $t \bar{t}$ production cross sections and rates in a one month $\left(10^{6} \mathrm{~s}\right)$ $A A$ run are also given.

\begin{tabular}{lcccc}
\hline \hline Ion Species & $\begin{array}{c}\sqrt{S_{N N}} \\
(\mathrm{TeV})\end{array}$ & $\begin{array}{c}p A \text { Luminosity } \\
\left(\mathrm{pb}^{-1} \mathrm{~s}^{-1}\right)\end{array}$ & $\begin{array}{c}\sigma(p A) \\
(\mathrm{pb})\end{array}$ & $\begin{array}{c}\text { Rate } \\
\text { (per month) }\end{array}$ \\
\hline Oxygen & 9.9 & $3.8 \times 10^{-4}$ & 2.9 & 1100 \\
Lead & 8.8 & $7.3 \times 10^{-7}$ & 55 & 40 \\
\hline \hline
\end{tabular}

TABLE II. Center of mass energies per nucleon-nucleon collision, $\sqrt{S_{N N}}$, and luminosities for $p A$ interactions at the LHC. The $t \bar{t}$ production cross sections and rates in a $10^{6} \mathrm{~s} p A$ run are also given. 


\section{FIGURES}

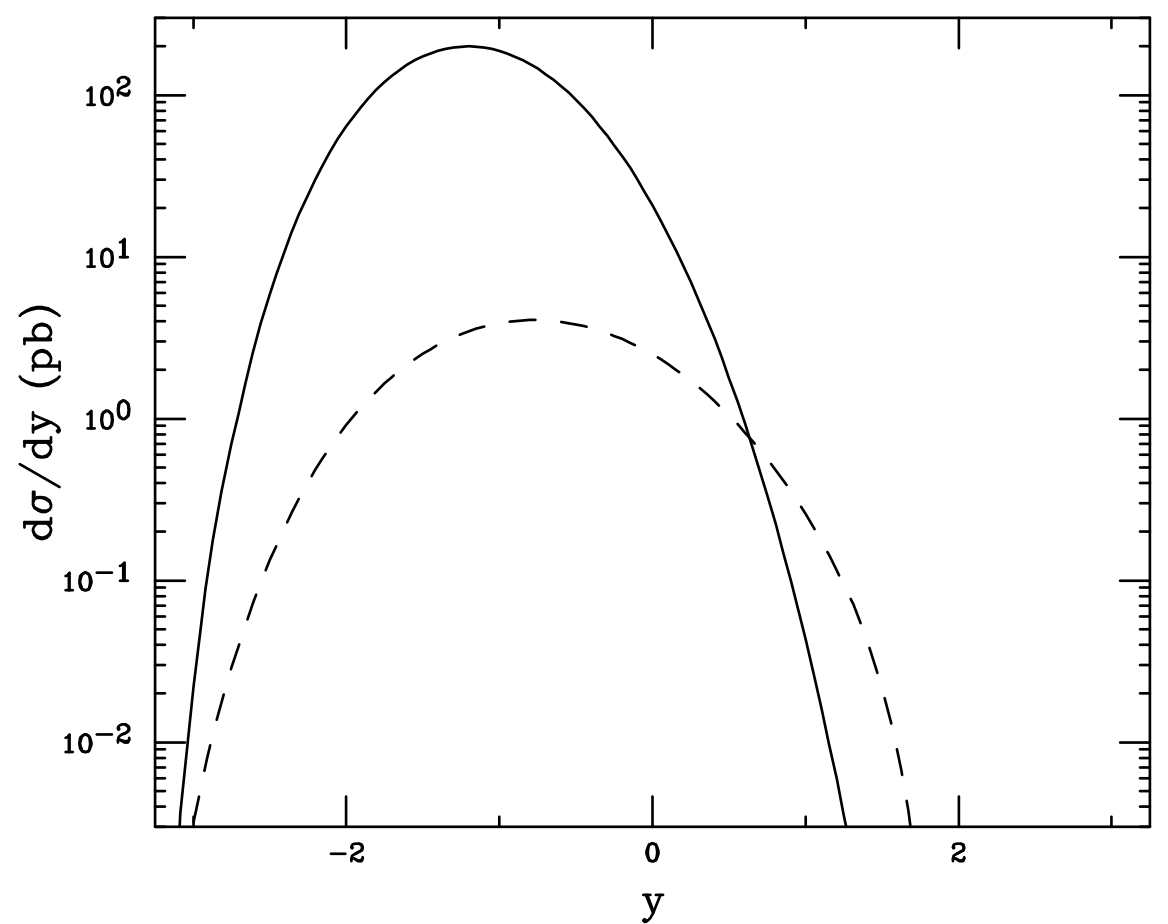

FIG. 1. The top quark rapidity distribution, $d \sigma / d y$, for photoproduced top at the LHC. The solid curve is for $\mathrm{Pb}+\mathrm{Pb}$ collisions while the dashed curve is for $\mathrm{O}+\mathrm{O}$ collisions. In this plot, the photon is emitted by the nucleus with positive rapidity. 


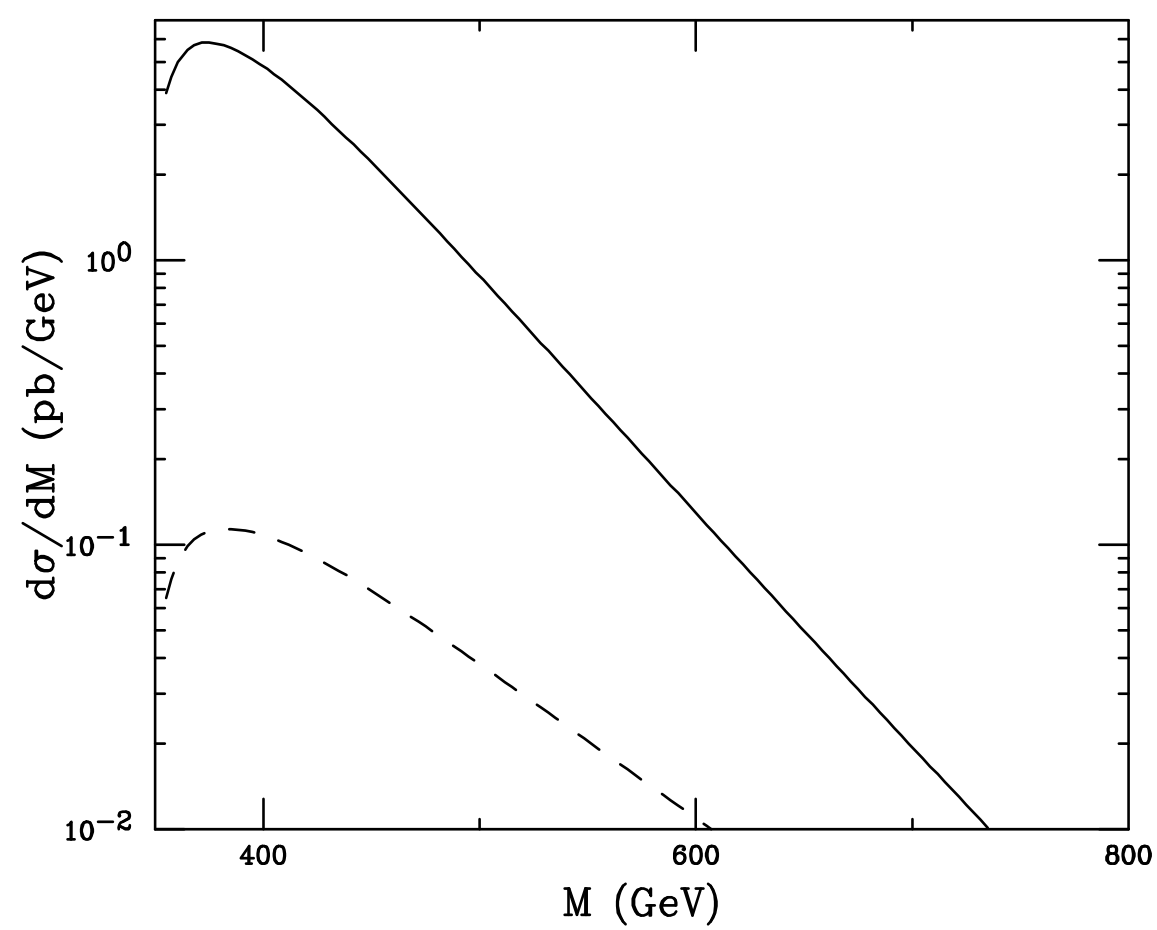

FIG. 2. The $t \bar{t}$ pair mass distribution, $d \sigma / d M$, for photoproduced top at the LHC. The solid curve shows the results for $\mathrm{Pb}+\mathrm{Pb}$ collisions while the dashed curve is for $\mathrm{O}+\mathrm{O}$ collisions. 